\title{
On the Alkaloid of Fritillaria verticillate WILD. var. Thunbergii BAKER. II. ${ }^{1)}$ The Structure of Verticine. ${ }^{* 1}$
}

We wish to provide herein the evidence which permits the assignment of the structure $(\mathrm{I})$ for verticine, ${ }^{* 2,1,2)}$ the main alkaloid of Fritillaria verticillata var. Thunbergii,*3 together with some modification of the partial structure proposed previously.

We have shown the presence of two secondary and a tertiary hydroxyls in $I, * 4$ diacetate (II), $\mathrm{C}_{31} \mathrm{H}_{49} \mathrm{O}_{5} \mathrm{~N}$, $* 5$ m.p. $117 \sim 120^{\circ}$, by successive oxidation of $\mathrm{I}$ to verticinone (III), ${ }^{1)}$ acetate (IV), $\mathrm{C}_{29} \mathrm{H}_{45} \mathrm{O}_{4} \mathrm{~N}$, m.p. $173 \sim 174^{\circ}$, and to verticindione (V). ${ }^{1)}$ Although V, and also III, were converted to I by sodium in ethanol or lithium in liquid ammonia in the presence of methanol, the reduction of both ketones with sodium borohydride, lithium aluminum hydride or aluminum isopropoxide as well as by catalytic reduction (Pt, ethanol) resulted in the formation of isoverticine (VI), $\mathrm{C}_{27} \mathrm{H}_{45} \mathrm{O}_{3} \mathrm{~N}$, m.p. 206 214 ${ }^{\circ}$, $\mathrm{pKa}^{\prime}$ 9.4; diacetate (VII), $\mathrm{C}_{31} \mathrm{H}_{49} \mathrm{O}_{5} \mathrm{~N}$, m.p. $124^{\circ}$. Since both $\mathrm{I}$ and VI afforded $\mathrm{V}$ on chromic acid oxidation, they are epimeric at one of the secondary hydroxyls. It was also assumed from the mode of formation in these reductions ${ }^{4,5)}$ that the two hydroxyls are both equatorial in I, while they are equatorial and axial in VI. This assumption was supported by the following observations: i) VI had a larger Rf values than I in paper and thin layer chromatography. ${ }^{5,6}$ ) ii) VI, contrary to I, was easily oxidized by two moles of $\mathrm{N}$-bromosuccinimide to develope a carbonyl band at $1705 \mathrm{~cm}^{-1} .^{7)}$ iii) I was

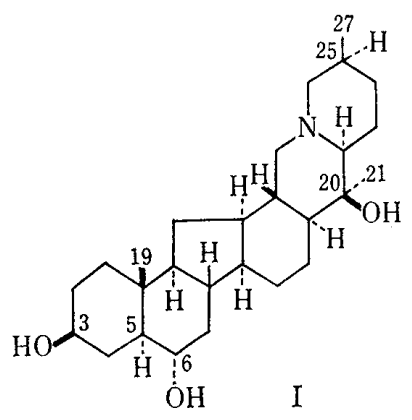<smiles></smiles>

III

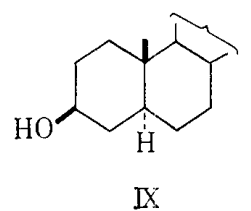<smiles>O=C1CCC23CCCC2CC(=O)CC3C1</smiles>

$\mathrm{V}$<smiles>[X]C1CCC2CCCC2(C)C12CCCC2C</smiles><smiles>CC12CCC(O)CC1CC1CCC2C1</smiles>

VI<smiles>O=C1CC2CC3CC(CC13)C21C=CCCC1</smiles><smiles>O=C1CCCC23CCCC2CCC1C3</smiles>

VIII<smiles>C=[N+]1CCCC(C)C1</smiles>

XII

*1 Presented at the 6th Symposium on Natural Product held at Sapporo on July 6th, 1962.

*2 The identity of I with peimine isolated by Chou and $\mathrm{Chen}^{3)}$ was established by the direct comparison carried out by Dr. J.H. Chu, Institute of Organic Chemistry, Academia Sinica, Shanghai, thus the presence of cevane skeleton being established in I. We are deeply indebted to Dr. Chu for his kind determination of the mixed melting point. We use the name "verticine" in favor of "peimine" in this paper, since the former has first been named by Fukuda") to the pure alkaloid, m.p. $223 \sim 224^{\circ}$. Elemental analyses of Fukuda's original sample agreed with the formula $\mathrm{C}_{27} \mathrm{H}_{45} \mathrm{O}_{3} \mathrm{~N}$, in our hand.

*3 For the historical aspect of the study on the constituents of Fritillaria species, see H. Morimoto, S. Kimata: This Bulletin, 8, 302, $871(1960)$, which also describe the isolation of peimine and its glucoside as well as some reaction of these compounds.

*4 UV: $\varepsilon_{210}^{\mathrm{EtOH}-\mathrm{HCl}} 10$. The value was erroneously printed in the reference 1.

$*_{5}$ All analytical values through this paper are in good agreement with the formulas shown.

1) Part I. This Bulletin, 9, 253 (1961).

2) M. Fukuda : Nippon Kagaku Zasshi, 50, 74 (1929); Sci. Repts Tohoku Univ., Ser. A, 18, 323 (1929).

3) T.Q. Chu, K. K. Chen : Chinese J. Physiol., 6, 265 (1932).

4) Cf. L.F. Fieser, M. Fieser : "Steroid" Reinhold Publ. Corp., 1959, p. 268 and references there cited.

5) D.H. R. Barton, G. A. Morrison in “Fortschritte der Chemie organischer Naturstoffe” Ed. by L. Zechmeister, Springer Verlag, 1961, p. 165 and the references there cited.

6) V. Cerny, J. Joska, L. Labler : Coll. Czech. Chem. Commun., 26, 1658 (1961).

7) Cf. L. F. Fieser, S. Rajagopalan, J. Amer. Chem. Soc., 71, 3938 (1949). 
the predominant component in the equilibrated mixture (sodium butoxide in butanol) both from I and VI. ${ }^{8)}$

The hydroxyl, which is thermodynamically more stable and more resistant to chromic acid, has been shown $\mathrm{n}^{1)}$ to be located at $\mathrm{C}-3$ of the cevane ring system, since, among other indications, dehydrodeoxoverticinone (VIII), derived from III via deoxoverticinone (IX), has a positive Cotton effect. The $\beta$-configuration of this hydroxyl was also supported by the sharp and simple band at $1242 \mathrm{~cm}^{-1}$ in the infrared spectrum of deoxoverticinone acetate, $\mathrm{C}_{29} \mathrm{H}_{47} \mathrm{O}_{3} \mathrm{~N}$, m.p. $183^{\circ} .{ }^{\text {. }}$ )

The other secondary hydroxyl is located at $\mathrm{C}-6$ because i) both $\mathrm{V}$ and cholestane3,6-dione underwent aerial oxidation in alkaline medium to develope an ultraviolet maximum ( $\nu_{\max }^{\mathrm{MeOH}-\mathrm{NaOH}} 375 \mathrm{~m} \mu$ ) due to the presense of a 4-ene-3,6-dione system in the product, ii) on heating with polyphosphoric acid, III yielded a cisoid $\alpha, \beta$-unsaturated

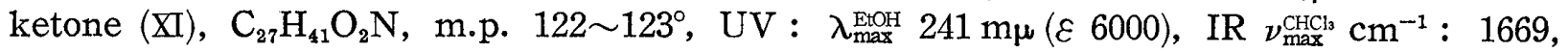

Table I. Chemical Shift of Methyl Signals

\begin{tabular}{|c|c|c|c|c|c|c|c|}
\hline \multirow{2}{*}{ Partial structures } & \multirow{2}{*}{$\mathrm{R}$} & \multicolumn{4}{|c|}{ Verticine series $^{a)}$} & \multicolumn{2}{|c|}{$5 \alpha-$ Steroid $^{11)}$} \\
\hline & & No. & 27-Me & 21-Me & $19-\mathrm{Me}(\mathrm{A})$ & $19-\mathrm{Me}(\mathrm{B})$ & $\delta_{A}-\delta_{B}$ \\
\hline & & $\mathrm{X}$ & $8.94(8)$ & 9.01 & 9.20 & $9.22^{b)}$ & -0.02 \\
\hline & & IX & $8.92(7)$ & 8.97 & 9.20 & 9.20 & 0.00 \\
\hline & & VII & $8.93(6)$ & 9.00 & 9.00 & $8.98^{b)}$ & +0.02 \\
\hline & $\int \mathrm{H}$ & I & $8.93(7)$ & 8.98 & 9.19 & & $-0.02^{c)}$ \\
\hline & Ac & П & $8.93(6)$ & 8.96 & 9.09 & & $-0.04^{d)}$ \\
\hline & $\{\mathrm{H}$ & $\mathrm{VI}$ & $8.91(7)$ & 8.96 & 8.96 & 8.98 & -0.02 \\
\hline & Ac & VII & $8.94(7)$ & 8.99 & 8.99 & 8.99 & 0.00 \\
\hline & $\mathrm{H}$ & III & $8.94(6)$ & 8.99 & 9.24 & $9.21^{a)}$ & +0.03 \\
\hline & Ac & IV & $8.97(7)$ & 9.01 & 9.24 & 9.22 & +0.02 \\
\hline & & V & $8.96(6)$ & 9.01 & 9.07 & $9.05^{b)}$ & +0.02 \\
\hline & & & $\begin{array}{r}8.94 \pm 0.03 \\
(6 \sim 8\end{array}$ & $8.99 \pm 0.03$ & & & \\
\hline
\end{tabular}

a) Measured at $60 \mathrm{Mc}$./sec. in $\mathrm{CDCl}_{3}$ and expressed in $\tau$-values. Figures in parentheses denote coupling constant in $\mathrm{c} / \mathrm{s}$.

b) Mean values.

c) Derived by subtracting the value $(9.20)$ for $5 \alpha$-pregnan-3 $\beta$-ol ${ }^{11}$ ) and the effect $\left.(+0.01)^{12}\right)$ of $6 \alpha$-hydroxyl from the value for verticine (9.19).

d) Derived by subtracting the mean value (9.17) for $5 \alpha$-steroidal $3 \beta$-acetate ${ }^{11}$ and the effect $\left(0.04^{12)}\right)$ of $6 \alpha$-acetoxyl group from the value for verticine diacetate (9.09).

8) Cf. D. H. R. Barton : J. Chem. Soc., 1953, 1027.

9) Cf. A. R.H. Cole, R. N. Jones, K. Dobriner, J. Amer. Chem. Soc., 74, 5571 (1952). 
1613 (vs), and iii) heating with one mole of hydrazine hydrate converted $\mathrm{V}$ to a dihydropyridazine derivative, $\mathrm{C}_{27} \mathrm{H}_{43} \mathrm{ON}_{3}, \mathrm{~m} . \mathrm{p} .>300^{\circ}$, IR $\nu_{\max }^{\text {Nujol }} \mathrm{cm}^{-1}: 3448,1631 .^{10)}$

Nuclear magnetic resonance spectral analysis confirmed the conclusion derived from the reactions described above. Chemical shifts for all the methyls in verticine and its derivatives are listed in Table I. For comparison, the Table also shows the 19-methyl signals in the $5 \alpha$-steroid series ${ }^{11)}$ and the differences between the corresponding chemical shifts in the two series.

As shown in the Table, each of the verticine derivatives exhibited three methyl signals consisting of one doublet and two singlets. The doublet and one of the singlets remain constantly in the range of $8.94 \pm 0.03 \tau$ and $8.99 \pm 0.03 \tau$ respectively, showing that these methyls are remote from any of the secondary hydroxyls. The position of the other singlet, on the other hand, is in the wider range $(8.96 \sim 9.24 \tau)$, depending on the nature of the substituents at $\mathrm{C}-3$ and $\mathrm{C}-6$, and the following shifts are observed; the down-field shift due to 3-ketone in VIII compared with the corresponding deoxo-compound, bisdeoxoverticindione $(\mathrm{X}), \mathrm{C}_{27} \mathrm{H}_{45} \mathrm{ON}, \mathrm{m} . \mathrm{p} .121 \sim 122^{\circ}$, IR $\nu_{\max }^{\text {Nujol }} \mathrm{cm}^{-1}: 3500,1125$, obtained by the Huang Minlon reduction of $\mathrm{V}$; and that due to the 1,3-diaxial disposition of two groups $^{11,12)}$ in VI compared with the epimeric (I); and the up-field shift due to 6-ketone in III and IV compared with IX. ${ }^{11)}$ These characteristic changes in the chemical shifts, as well as the coincidence of all of the methyl signals of the corresponding members in both series, established the partial stereochemistry of I to be $5 \alpha$-cevane- $3 \beta, 6 \alpha$-diol.

The tertiary hydroxyl group, shown by the bands near 3500 and $1125 \mathrm{~cm}^{-1}$ in infrared spectra of $I I, I V, V$, VII, VIII, and $X$, must be at either $\mathrm{C}-20$ or $\mathrm{C}-25$ because only one methyl signal appears as a doublet in the nuclear magnetic resonance spectra of all compounds examined (Table I). Of the two possible sites, C-25 was ruled out because i) I, V, and VI had comparable basisity with cevine $\left(\mathrm{pKa}^{\prime} 9.48^{13)}\right)$, and ii) mass spectra of I, V, VI, and VII always displayed as a base peak the ion, $112 \mathrm{~m} / \mathrm{e}$, attributed to XII, besides their respective molecular ions. That the configuration of the tertiary hydroxyl is axial, as in cevine, ${ }^{13}$ ) was implied by the basisity and deduced from the infrared spectrum of $\mathrm{X}\left(\mathrm{in} \mathrm{CCl}_{4}\right.$ ) which showed only an intramolecularly hydrogen-bonded $\mathrm{OH}$ band at $3540 \mathrm{~cm}^{-1}$ (concentration-independent in $0.1 \sim 0.01 \mathrm{M}$ ). ${ }^{14}$ )

The stereochemistry of C-27 methyl was concluded to be axial from the chemical shifts and coupling constants of the doublets (Table I) which were quantitatively in accordance with those of cevine derivatives. ${ }^{15}$ )

The evidence given so far confirms structure (I) for verticine but with the stereochemistry at positions $\mathrm{C}-8, \mathrm{C}-9, \mathrm{C}-12, \mathrm{C}-14, \mathrm{C}-16$, and $\mathrm{C}-17$ undetermined; however by analogy with the steroid series and from biogenetic considerations it is suggested that the stereochemistry at these positions is as depicted in $I$.

The optical rotatory dispersion curve of ${ }^{1 I},{ }^{1)}$ which is strikingly similar to that of 7-oxocholestanyl acetate ${ }^{16)}$ and therefore is responsible for the erroneous proposal in our previous paper, ${ }^{1)}$ was not essentially changed by the addition of acid, showing a lar ge hindrance to ketal formation. The difference between the shape of the curves of III and 6-oxocholestanyl acetate ${ }^{16}$ ) may be attributed to a change in conformation of ring $\mathrm{B}$ due to the size of the ring $\mathrm{C}$ in these compounds.

10) Cf. R.E. Marker, E. Rohrmann : J. Amer. Chem. Soc., 61, 946 (1939),

11) R.F. Zürcher : Helv. Chim. Acta, 44, 1380 (1961).

12) Y. Kawazoe, Y. Sato, M. Natsume, H. Hasegawa, T. Okamoto, K. Tsuda : This Bulletin, 10, 338 (1962).

13) O. Jeger, V. Prelog in “The Alkaloids” Ed. by R.H.F. Manske, Academic Press, Vol. 3, 1953, p. 247 : Vol. 7, 1960, p. 363.

14) Cf. W.A. Ayer, G. G. Iverach : Tetrahedron Letters, 1962, 87.

15) S. Itô, S. M. Kupchan, J. B. Stothers : In preparation.

16) C. Djerassi, W. Closson, A. E. Lippman : J. Amer. Chem. Soc., 78, 3163 (1956). 
Our deep gratitude is expressed to Mr. M. Kodama, Tohoku University, for his cooperation in some experiments, to Prof. J. B. Stothers, University of Western Ontario, for NMR measurement, and to Prof. C. Djerassi, Stanford University, for mass spectra.

\begin{tabular}{lll} 
Department of Chemistry, & Shô Itô & (伊東 椒) \\
Tohoku University, & Michiharu Kato（加藤紀元） \\
Sendai. & Koichi Shibata (柴田耕一) \\
\multicolumn{2}{l}{ Received June 25, 1963 } & Tetsuo Nozoe (野副鉄男)
\end{tabular}

Revised July 23, 1963

$\left[\begin{array}{l}\text { Chem. Pharm. Bull. } \\ 11(10) \text { 1340 } ~ 1342\end{array}\right]$

UDC $547.918: 615.711 .5$

\section{Hydroxylation of Digitoxigenin Derivatives by Absidia orchidis}

In the previous paper ${ }^{1)}$ we reported the hydroxylation of digitoxigenin $(I)$ at $1 \beta, 5 \beta$, and $7 \beta$ positions by Absidia orchidis (VuILl.) HAGEM, a microorganism known to hydroxylate progesterone (II) at $6 \beta, 7 \alpha$, and $11 \alpha,{ }^{2)}$ and Reichstein's substance $\mathrm{S}$ (III) at $6 \beta$, $11 \alpha$, and $11 \beta$ positions. ${ }^{3)}$ In order to investigate the relationships between the structure of substrate molecules and the positions to be hydroxylated, we carried out the trans-

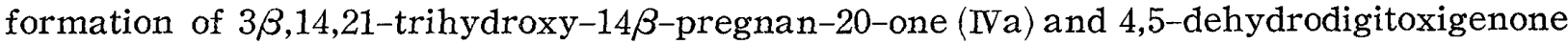
(V) using this microorganism with the results that the former compound is hydroxylated at $1 \beta$ and the latter mainly at $7 \beta$ and $12 \beta$ positions.

$A$. orchidis was grown for 66 hours with shaking on a nutrient medium containing glucose, peptone and corn steep liquor, the mycelium harvested, washed and suspended in distilled water. The substrate (IVb) dissolved in methanol was added to this mycelium suspension and incubation was continued for a further 48 hours. After usual treatment of the fermentation filtrate, a monohydroxylated product (VIa), $\mathrm{C}_{21} \mathrm{H}_{34} \mathrm{O}_{5}, \mathrm{~m} . \mathrm{p}$. $252 \sim 263^{\circ}$, was obtained. As this substance gave a triacetate (VIb), $\mathrm{C}_{27} \mathrm{H}_{40} \mathrm{O}_{8}$, m.p. 158 $162^{\circ},[\alpha]_{\mathrm{D}}^{25}-0.4^{\circ}$ (pyridine), the newly introduced hydroxyl was considered to be a secondary or a primary one, and proved to be at $1 \beta$-position on identification of $\mathrm{VIb}$ with ketol triacetate derived from acovenosigenin A (VII).

While the usual bioconversion performed by us of 4,5-dehydrodigitoxigenone (V) with $A$. orchidis produced several kinds of product, the use of suspensions of mycelium preincubated with progesterone ${ }^{4)}$ gave one of them predominantly. This compound (IXa), $\mathrm{C}_{23} \mathrm{H}_{30} \mathrm{O}_{6}$, m.p. $287 \sim 290^{\circ},[\alpha]_{D}^{25}+66.7^{\circ}$ (pyridine), UV : $\lambda_{\max }^{\mathrm{EOH}} 226 \mathrm{~m} \mu^{* 1}(\log \varepsilon 4.32)$, gave a diacetate $(\mathrm{IXb}) \mathrm{C}_{27} \mathrm{H}_{34} \mathrm{O}_{8}$, m.p. $267 \sim 268^{\circ},[\alpha]_{0}^{2}+52.1^{\circ}$ (pyridine), showing that both of the newly introduced hydroxyls are secondary or primary. Treatment of IXa with 1\% hydrochloric acid in acetone afforded a $\Delta^{4,6}-3$-ketone $(\mathrm{X}), \mathrm{C}_{23} \mathrm{H}_{28} \mathrm{O}_{5}$, m.p. $279 \sim 283^{\circ}$, UV: $\lambda_{\max }^{\text {Erof }} 283 \mathrm{~m} \mu(\log \varepsilon 4.40)$. This fact and the unsuccessful reduction of IXa with zinc dust in acetic acid indicated that one of the hydroxyls introduced into ring $B$ was at 7 -position

*1 The appearance of absorption band at this wave length is due to overlapping of an absorption for $\alpha, \beta$-unsaturated ketone at $241 \mathrm{~m} \mu$ and that of unsaturated lactone at $217 \mathrm{~m} \mu$.

1) Y. Nozaki, T. Okumura: Agr. Biol. Chem., 25, 515 (1961); Y. Nozaki : Ibid., 25, 884 ; 879 (1961); H. Ishii, Y. Nozaki, T. Okumura, D. Satoh: This Bulletin, 11, 156 (1963).

2) Y. Nozaki, H. Ishii, T. Okumura : Collection of Lectures Commemorating the Inauguration of the New Shionogi Research Laboratory Building, p. 227 (1963).

3) Y. Nozaki, E. Masuo, H. Ishii, T. Okumura, D. Satoh : Ann. Rept. Shionogi Research Lab., 11, 9 (1961); O. Hanc, A. Capek, B. Kakac : Folia Microbiologica, 6, 392 (1961).

4) Y. Nozaki, E. Masuo, D. Satoh : Agr. Biol. Chem., 26, 399 (1962). 